\title{
The Determination of Surface Tension of Traditional Chinese Medicine Tablet
}

\author{
Xiaolu Zhang, Hao Zhang, Ke Liang, Er’jia Zhu, Ruisi Yan, Hui Yang, Zaiyou Tan* \\ School of Pharmacy, Guangdong Pharmaceutical University, Guangzhou, China \\ Email: tanzaiyou@medmail.com.cn
}

Received September 27, 2012; revised October 26, 2012; accepted November 4, 2012

\begin{abstract}
In this paper we aimed to determine the surface tension of traditional Chinese medicine (TCM) tablet and tried to provide a key reference parameter to control tablet film coating quality. Using contact angle tester, the Chinese medicine tablet, a famous Fufang Danshen tablet CP as a model example, was determined by Zisman critical surface tension method. Our study showed that the critical surface tension of Fufang Danshen tablet CP was determined to be 18.00 dyne $/ \mathrm{cm}$. The Zisman method, hence, can determine successfully the critical surface tension of Chinese medicine tablet and will become an effective manner to control tablet film coating quality.
\end{abstract}

Keywords: Surface Tension; Contact Angle; The Zisman Critical Surface Tension Method; Fufang Danshen Tablet CP; Chinese Medicine

\section{Introduction}

Film coating is a process which involves the deposition of a membrane consisting of polymer, plasticizer, colourant and possibly other additives onto the surface of a pharmaceutical dosage form, typical a tablet or a granule. Over the past two decades, there has been a dramatic increase in the use of this process in China. The reasons for file coating are many and varied. They include [1]: 1) To improve product appearance, odour and taste and to aid swallowing. 2) To aid identification and hence decrease the risk of confusion, especially when patients have to take several preparations. 3) To protect the active ingredient against heat, light and moisture. 4) To separate incompatible active ingredients present in the preparation. 5) To prevent dust formation during subsequent packing on high-speed packing lines. 6) To control the release of an active ingredient by use of either a coating with $\mathrm{pH}$-dependence solubility or a coating that acts as a diffusion membrane. Compared to the conventional sugar coat, the film coat is relatively thin typically $10-100 \mu \mathrm{m}$. Although the technology involved in the application of such a thin coating to a substrate is not new, having precedents in both the paints and adhesive technologies, problems do occur resulting in a number of film defects. These can either affect the visual appearance of the coated tablet or, more importantly, result in the loss of continuity of the film and thus affect the release of the active ingredient from the preparation. Therefore, look-

\footnotetext{
"Corresponding author.
}

ing for the reference factor of quality control of film coating of tablet is necessary.

One of the requirements of tablet film coating is that good adhesion of the coat to the tablet must be achieved. The properties of the coating formulation as well as those of the tablet can influence adhesion. The prerequisite for good adhesion is the spreading of the atomized droplets over the surface of the tablet and limited penetration of the coating solution into pores of the tablet. Both of these are controlled by the surface tension of the tablet and the coating. The surface tension of solid surface can be determined using: Owen's method [2], Van Oss's [3] method and Zisman's method [4]. The both of Owen's and Van Oss's must depend on accurate interaction energy parameters of all components to determine the surface tension of TCM tablet but it is difficult owing to the ingredients of the tablet extracting from various herbs are complicate. So we make an experiment in order to find out the surface tension of CTM tablet by Zisman's method.

Zisman and his associates found that when the cosine of the contact angle, was plotted versus the surface tension for a homologous series of liquids spread on a surface such as Teflon (polytetrafluoroethylene), a straight line resulted. The line can be extrapolated to $\cos \theta=1$, that is, to a contact angle of zero, signifying complete wetting. The surface tension at $\cos \theta=1$ was given the term critical surface tension and the symbol $\gamma_{c}$, Zisman concluded that $\gamma_{c}$ was characteristic for each solid. We should try to find out such $\gamma_{c}$ of film coating of TCM 
tablet as a characteristic parameter to control tablet coating quantity. In this paper, we will report the traditional Chinese medicine tablet, a famous Fufang Danshen tablet $\mathrm{CP}$ [5] as a model example, was determined using contact angle tester by Zisman critical surface tension method.

\section{Instruments and Reagents}

\subsection{Instruments}

The JZ8002 electronic balance (Shanghai Balance Instrument Factory); GZX-9240MBE significant number of blast oven (Shanghai Xun Bo Industrial Co., Ltd. Medical Equipment Factory); JC2000C1 contact angle measuring instrument (Shanghai Zhong Chen Technology Co., Ltd.).

\subsection{Reagents}

Pure water (Guangdong Pharmaceutical University); Formamide (Sino Pharmaceutical and Chemical Reagent Co., Ltd., AR); Glycol (Guangzhou Chemical Reagent Factory, AR); Butanol (J \& K, AR).

\section{The Contact Angle Measurements}

\subsection{Preparation of Fufang Danshen Tablet CP and the Test Liquids}

We prepare the Fufang Danshen tablet CP by weighing the formulation $400 \mathrm{mg}$ and Repressing them into $2 \mathrm{~mm}$ thickness and smooth surface tablet for sparing according to Chinese Pharmacopoeia as a model tablet study and design several test liquids (pure water, formamide, glycol, butanol etc.) system.

\subsection{Measurements of Contact Angle of Fufang Danshen Tablet CP}

Pretreatment: Place $5 \mathrm{ml}$ water, Butanol, glycol, formamide in the Petri dish, respectively and then put the Petri dish in closed desiccators. The tablets employed to test were located in the closed desiccators for $48 \mathrm{~h}$ until the surface was saturated under the steam of the test liquid.

Measure: The measurements were conducted through the way of sessile drops with a computerized JC2000A contact angle goniometer (Shanghai Balance Instrument Factory). When the JC2000A contact angle goniometer was operated, the production of the sessile drops was yielded by performing the processes as follows:

1) Control the temperature to be $25^{\circ} \mathrm{C}$.

2) The infusion needle of the instrument was coated with paraffin to ensure that the droplet is always in the needle position.

3) Placing the needle near the surface, the corresponding volume of liquid was squeezed out of the syringe.
4) Squeezing a drop of tested liquid on the tablet surface, focusing camera $5 \mathrm{~s}$ latter.

5) Recorded every 5 seconds until 30 seconds and test 10 times for each test liquid Contact angle was measured using the JC2000A, which takes a video image of the drop and uses goniometer to determine and calculate.

6) Then select the mean value as the $\theta$.

Each measurement should be finished less than $1 \mathrm{~min}$ to minimize the effects of evaporation of the liquids. It was found experimentally that the contact angle remained constant within this operating time. An advancing contact angles was measured in this way. Each number in the Table 1 is the mean value of at least 10 single measurements. The results and images are shown in Figure 1.

\section{Results and Discussion}

To characterize the TCM tablet, the surface tensions of several liquids and their contact angles on the model Fufang Danshen tablet $\mathrm{CP}$ were determined at $25^{\circ} \mathrm{C}$, as showed in the following Table 1.

Plot $\cos \theta$ versus $\gamma$, they appear to fit nicely the Zisman principle, producing a straight line that extrapolates to $\cos \theta$ corresponding to a critical surface tension of $\gamma_{c}=$ $18.00 \mathrm{dyne} / \mathrm{cm}$ as shown in Figure 1. From the results obtained, the liquid in the tablet would be expected to the best wet on the model tablet. For a more exact calculation of the critical surface tension $\gamma_{c}$, least-squares linear regression analysis can be applied to yield:

$$
\cos \theta=-0.0029 \gamma_{c}+1.0522, R^{2}=0.9859 \text {. }
$$

According to the above critical surface tension of Fufang Danshen tablet CP which was tested to be 18.00 dyne $/ \mathrm{cm}$, we prepared 3 batches of film control release coated tablets of Fufang Danshen. The results of release rate and the release profiles of 3 batches of tablets were exhibited in the Table 2 and Figure 2.

The results showed that the released rate and the verification of 3 batches which were good agreement on the quality requirements of control drug release formulations.

\section{Conclusion}

Although one frequently desires to determine the relative

Table 1. Contact angles of liquids and surface tension of Fufang Danshen tablets $\mathrm{CP}$ at $25^{\circ} \mathrm{C}$.

\begin{tabular}{cccc}
\hline & Surface tension & Contact angle & $\cos \theta$ \\
\hline Water & 72.8 & 31.43 & 0.845 \\
Formamide & 58.2 & 28.4 & 0.876 \\
Glycol & 48 & 22.14 & 0.922 \\
Butanol & 24.6 & 11.6 & 0.98 \\
\hline
\end{tabular}


Surface Tension of FufangDanshen Tablet CP by Zisman Method

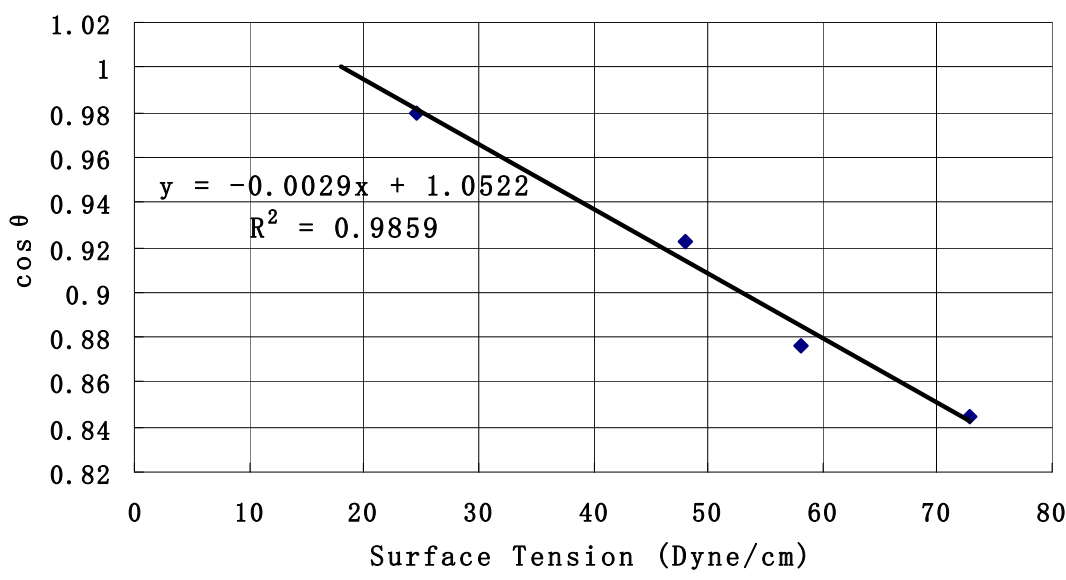

Figure 1. Surface tension of Fufang Danshen tablets CP by Zisman method.

Table 2. Drug release with different batches.

\begin{tabular}{cccc}
\hline Time (h) & 1 & 2 & 3 \\
\hline 2 & 21.4 & 22.7 & 19.5 \\
4 & 42.1 & 45.5 & 39 \\
6 & 57.3 & 62.1 & 54.2 \\
8 & 73.6 & 76.2 & 70.7 \\
10 & 83.4 & 84.9 & 79.2 \\
12 & 87.8 & 89.7 & 84.1 \\
\hline
\end{tabular}

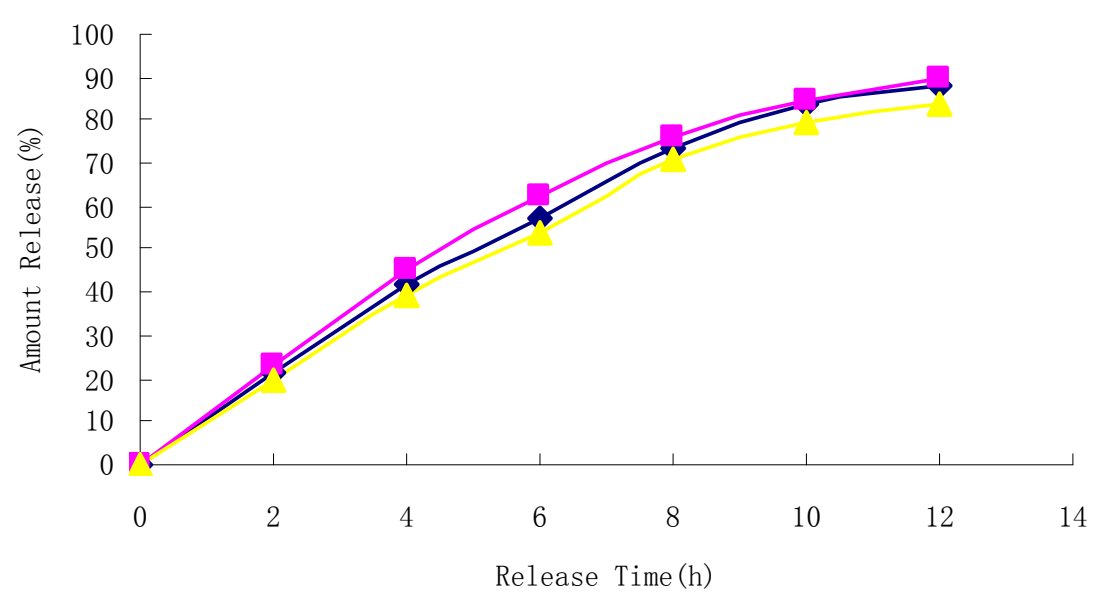

Figure 2. Release profile of verification test.

efficiencies of wetting agents, it is difficult to measure directly the contact angle of a solid surface. As a result of these difficulties, our experimental tests have indicated Zisman' method can be used to measure the critical surface tension of the TCM tablet in industry. To ensure the quantity control the TCM tablet, we suggest Zisman's method can be used for estimating the ability of a wetting agent or file coating on the surface of the tablet and the application property of such products are ordinary determined by subjective evaluation. More importantly, Zisman's method can be applied to the surface tension of solid which was constituted with various components. It is difficult for other indirect methods such as the Owen's method and Van Oss's method to determine the surface 
tension of solid owing to they must depend on accurate interaction energy parameters of each component. However, in order to ensure accessing accurate surface tension values, the nature of the surface materials should be poorly hygroscopic. Meanwhile, the humidity of experimental environment must be controlled. The surfaces of tested samples should be saturated by the tested liquid during experiment procedure. This job calls for patience.

\section{REFERENCES}

[1] D. Ganderton and J. Trevor, "Advances in Pharmaceutical Science," Academic Press, London, Vol. 6, 1992, pp. 6566.
[2] D. K. Owens and R. C. Wendt, "Estimation of the Surface Free Energy of Polymers," Journal of Applied Polymer Science, Vol. 13, No. 8, 1969, pp. 1741-1747. doi:10.1002/app.1969.070130815

[3] C. J. Van Oss, M. K. Chaudhury and R. J. Good, "Interfacial Lifshitz-van der Waals and Polar Interactions in Macroscopic Systems," Chemical Reviews, Vol. 88, No. 6, 1988, pp. 927-941. doi:10.1021/cr00088a006

[4] W. A. Zisman, "Contact Angle, Wettability and Adhesion in Advances in Chemistry Series," American Chemical Society, Vol. 9, No. 43, 1964, p. 513.

[5] "Chinese Pharmacopoeia," The Medicine Science and Technology Press of China, Beijing, 2010, pp. 904-905. 